\title{
Einige Anmerkungen zu berufsorientiertem und fachsprachlichem Deutschunterricht aus linguistischer und didaktisch-methodischer Sicht: Medizinische Fachsprache
}

\author{
Věra Janíková
}

\section{Einführung}

In den letzten Jahren hat die Nachfrage nach berufsorientiertem und fachsprachlichem ${ }^{1}$ Fremdsprachenunterricht auch an den tschechischen Schulen, Hochschulen und diversen Sprachkursen deutlich zugenommen. Man kann für diese Tendenz mehrere Gründe finden, die sich im Allgemeinen aus den jüngsten Entwicklungen im Fremdsprachenlernen sowie den veränderten Auffassungen über die Notwendigkeit von Fremdsprachenkenntnissen in einer modernen Gesellschaft ergeben. Denn eines der grundlegenden Prinzipien des heutigen Sprachunterrichts im europäischen Kontext lautet: Durch die bessere Kenntnis moderner europäischer Sprachen wird die Kommunikation und Interaktion zwischen Europäern verschiedener Muttersprachen erleichtert und dadurch die Mobilität und die Zusammenarbeit gefördert. Einen wichtigen Beitrag leistet in diesem Zusammenhang gerade der berufsorientierte und fachsprachliche Fremdsprachenunterricht. CHRIST $(2002,26)$ spricht in seinen Überlegungen zu den neuen Dimensionen im Fremdsprachenunterricht des 21. Jahrhunderts u. a. das ,lebensbegleitende' Lernen an, und der berufsvorbereitende, berufsbegleitende und fachsprachliche Fremdsprachenunterricht gehört zweifellos in diese Kategorie. Entsprechende Unterrichtsformen erfahren heute in fremdsprachlichen Veranstaltungen jeglicher Art einen wesentlichen Zuwachs.

Der berufsorientierte und fachsprachliche Deutschunterricht im tschechischen Kontext steht nicht abseits der europäischen Tendenzen, ist aber trotzdem durch gewisse Spezifika zu kennzeichnen, die aus den jüngsten Entwicklungen im schulischen sowie außerschulischen Fremdsprachenlernen in Tschechien abzuleiten sind. Während hier an allen Schultypen bis in die Hälfte der 90er Jahre des zwanzigsten Jahrhunderts Deutsch als erste Fremdsprache gelernt wurde, ist es heutzutage Englisch - genauso wie in vielen weiteren europäischen Ländern. Der Deutschunterricht folgt im Durchschnitt an zweiter Stelle. Die

1 Unter dem Begriff berufsorientierter Fremdsprachenunterricht verstehen wir in diesem Beitrag den Sprachunterricht an den Berufsschulen, wobei fachsprachlicher Fremdsprachenunterricht sich eher auf den hochschulischen studienbegleitenden Sprachunterricht bzw. auf diverse fachsprachlich orientierte schulische und außerschulische Sprachkurse bezieht. Aus linguistischer und sprachdidaktischer Sicht werden beide Begriffe synonym betrachtet, die Unterschiede sind in der Unterrichtspraxis eher in der methodisch-didaktischen Perspektive zu sehen. 
jüngsten statistischen Angaben zeigen, dass diese Situation vor allem für die Hauptschulen, Gymnasien, fachorientierten Mittelschulen und Hochschulen (als studienbegleitender Sprachunterricht) charakteristisch ist, wobei an den meisten Berufsschulen Deutsch zur Zeit eine Vorrangstellung einnimmt. Die Gründe dafür sind verschieden, von geographischen und ökonomischen bis hin zu historischgesellschaftlichen.

\section{Ziele des berufsorientierten und fachsprachlichen Unterrichts und die Lehrerrolle}

Eine prägnante Festlegung der Ziele des berufsorientierten und fachsprachlichen Fremdsprachenunterrichts findet man bei BUHLMANN und FEARNS (2000), die sagen, dass Sinn und Zweck des Fachsprachenunterrichts darin bestehe, „den Lerner in seinem Fach sprachlich handlungsfähig zu machen bzw. ihm den Erwerb der sprachlichen Handlungsfähigkeit in seinem Fach zu ermöglichen, zumindest aber zu erleichtern. Unter sprachlicher Handlungsfähigkeit im Fach verstehen wir die Fähigkeit des Lerners, sich in der Zielsprache (L2) angemessen zu informieren und zu verständigen. Angemessen bedeutet in diesem Zusammenhang, daß der Lerner in der Lage ist, mit seinen sprachlichen Mitteln unter Nutzung von Arbeitsstrategien Texten Maximum an Informationen zu entnehmen. Angemessene Verständigung bedeutet, daß sich der Lerner auf der Wissensstufe, auf der er sich gerade befindet, eindeutig und sachlich ausreichend differenziert äußern kann" (BUHLMANN/ FEARNS: 2000, 7).

Die Lehrkräfte, die eine allgemeinsprachliche Ausbildung samt der sprachdidaktischen Vorbereitung abgeschlossen haben und Deutsch als Berufsoder Fachsprache unterrichten sollen, geraten oft in Unsicherheit von zweierlei Art: Einerseits müssen sie sich mit dem konkreten Beruf/Fach und seiner entsprechenden Terminologie vertraut machen, andererseits - aus methodisch-didaktischer Sicht - effiziente Methoden wählen sowie ausgewogene und relevante Ziele im Spannungsfeld zwischen allgemeinsprachlichem und berufs- oder fachsprachlich orientiertem Unterricht festlegen. Man kann die Schwierigkeiten dieser Lehrenden wie folgt zusammenfassen:

- Der Lehrer ist meist kein Fachmann auf dem Gebiet, dessen Sprache er gerade vermitteln soll.

- Der Lehrer hat in der Regel keine Ausbildung als Fachsprachenlehrer erhalten.

- Der Lehrer stößt bei dem Versuch, sich einzuarbeiten, auf linguistische, methodische und didaktische Informationen und Aufsätze, die für ihn in ihrer Fülle schwer überschaubar und in ihrer Bedeutung für den Unterricht nicht leicht zu beurteilen sind. 
- Der Lehrer sieht sich oft der Situation gegenüber, dass er einen Fachsprachenkurs/fachsprachlichen oder berufsorientierten Unterricht geben muss, für den er kein oder kein ausreichendes oder befriedigendes Material zur Verfügung hat (vgl. BUHLMANN/FEARNS: 2000, 8).

Eine der wichtigsten Hilfestellungen für die Lehrenden wäre zweifellos die Integration der berufsorientierten und fachsprachlichen Fremdsprachenvermittlung in die fremdsprachendidaktischen Curricula der universitären Lehrerausbildung und -fortbildung, wobei diese Integration zur Zeit noch unter gewissen Defiziten leidet. Auch aus diesem Grund widmet sich der folgende Beitrag einigen linguistischen Aspekten und bringt verschiedene sprachdidaktische Anmerkungen, die mit praktischen Empfehlungen für die fachsprachliche Unterrichtspraxis versehen sind.

\section{Linguistische Besonderheiten der Fachsprache}

Aus sprachdidaktischer Sicht scheint es effizient zu sein, wenn der Fachsprachenlehrer die fachsprachlichen Merkmale reflektiert und mit ihnen im Unterricht gezielt arbeitet (vgl. BUHLMANN/FEARNS: 2000). Ein Hauptmerkmal der Fachsprache liegt in der gehäuften Verwendung von Fachwörtern bzw. Fachbegriffen, ${ }^{1}$ die in der allgemeinen Sprache selten gebraucht werden. Die Fachsprache verwendet jedoch gleichzeitig keine Strukturen, die nicht auch in der allgemeinen Sprache vorkommen. Sie unterscheidet sich allerdings von der Alltagssprache durch die Häufigkeit, mit der gewisse morphologisch-syntaktische Strukturen sowie lexikalische Phänomene vorkommen, die Schwierigkeiten im Verständnis und in der Verwendung bereiten können.

\section{a) Lexikalisch-morphologische ${ }^{2}$ Besonderheiten}

Aus Sicht der Morphologie in ihrer grammatischen und formalen Funktion und der fachsprachlichen Syntax kann man in den Fachsprachen v. a. folgende Spezifika identifizieren:

- häufiger Gebrauch des substantivierten Infinitivs (das Hobeln),

- das Vorherrschen des Indikativs als Modus für die Allgemeingültigkeit,

- der häufigste Gebrauch des Präsens,

- sehr häufiges Vorkommen von Passivformen,

- eine relativ häufige Verwendung der Genitivform bei Substantiven,

1 Nach BUHLMANN/FEARNS $(2000,33)$ hat der Fachbegriff als Denkelement des Faches einen bestimmten Inhalt, der die Gesamtheit seiner Merkmale repräsentiert (Glühlampe = Lampe + lichtaussendender fester Stoff + lichtaussendend infolge Erhitzens/Stromwärme). In dieser Hinsicht wird auch der Begriff Fachwort verwendet (siehe weiter unten).

2 Im Bereich der Morphologie der Fachsprachen sind zwei Komponenten besonders wichtig, und zwar die Formbildung und Wortbildung (BUHLMANN/FEARNS: 2000, 16). 
- semantisch notwendige Verwendung von Präpositionen (Präzision, Differenzierung und Sprachökonomie),

- häufige Verwendung von Adjektiven auf -bar, -los, -reich, -arm, -frei, -fest usw. (brennbar, nahtlos, vitaminreich, sauerstoffarm, rostfrei) und Adjektiven mit dem Präfix nicht (nicht leitend, nicht rostend),

- sehr häufige Verwendung von mehrgliedrigen Komposita,

- häufiges Vorkommen on Zusammensetzungen mit Ziffern, Buchstaben, Sonderzeichen (60-Watt-Lampe) und Mehrwortkomplexen (elektronische Datenverarbeitung),

- Bildungen mit und aus Eigennamen (röntgen, Ottomotor),

- fachspezifische Abkürzungen (DGL für Differenzialgleichung).

b) Syntaktische Besonderheiten

- häufiger Gebrauch von Funktionsverbgefügen (in Betrieb nehmen),

- unpersönliche Ausdrucksweise (man nimmt dazu, Strablungen lassen sich schwer nachweisen),

- unpersönliche Darstellungen (die 3. Person Sg. herrscht als Ausdruck der Beobachtung von Zuständen, Abläufen und Erscheinungen vor),

- Verwendung von Nominalisierungen,

- Verwendung von erweiterten Nominalphrasen anstelle von Gliedsätzen (nach der theoretischen Vorklärung) (vgl. ROELCKE: 1999; BUHLMANN/ FEARNS: 2000; JANÍKOVÁ: 2006).

\subsection{Das Fachwort und seine Eigenschaften}

Für sprachdidaktische Zwecke halten wir den Begriff Fachwort für besonders günstig. Diesen Begriff verwendet z. B. ROELCKE (1999), der auch die grundlegenden Eigenschaften eines ,Fachworts' transparent skizziert. Er definiert das Fachwort als die kleinste bedeutungstragende und zugleich frei verwendbare sprachliche Einheit, die innerhalb einer Kommunikation eines bestimmten menschlichen Tätigkeitsbereiches gebraucht wird.

\section{a) Exaktheit}

Unter Exaktheit ist im Allgemeinen ein möglichst adäquater Bezug fachsprachlicher Ausdrücke zu den Gegenständen, Sachverhalten und Vorgängen des betreffenden menschlichen Tätigkeitsbereiches zu verstehen.

\section{b) Eindeutigkeit}

Eindeutigkeit von Fachwörtern zielt auf das Verhältnis von fachlichen Ausdrücken und Bedeutungen ab. Den systemlinguistischen Erkenntnissen der Fachsprachenforschung nach weisen Fachwörter eines Fachwortschatzsystems genau eine Bedeutung auf (Monosemie). 


\section{c) Vagheit (Mehrdeutigkeit)}

,Vagheit' ist hier als Synonym für fachsprachlich Polysemie und Synonymie zu verstehen.

- Für das Wort Edelgasgleichrichterröhre sind z. B. mindestens sieben weitere Synonyme bekannt: Gasgleichrichterröhre, gasgefüllter Katodengleichrichter, gasgefüllte Diode, Gasdiode, Glimmdiode, Glimmröhre.

- Bei Kant lassen sich z. B. beim Wort Vernunft neunzehn verschiedene Bedeutungen unterscheiden (philosophischer Fachwortschatz).

Einige weitere Ursachen für systematische Mehrdeutigkeit bei Fachwörtern (neben assoziierenden Bedeutungsvariationen) sind folgende:

- Der steigende Benennungsbedarf innerhalb einzelner Fächer, der eine vielfältige Verwendung von bereits bestehenden Einheiten des Wortschatzinventars erforderlich macht.

- Innenfachliche Meinungsvielfalt, bei der bereits eingeführte Fachwörter unter verschiedenen Voraussetzungen unterschiedlich interpretiert werden.

- Fachsprachliche Interferenzen, die zu einem Gebrauch bestimmter Fachwörter in verschiedenen Fächern mit jeweils unterschiedlichen Bedeutungen führen.

\section{d) Fachsprachliche Metaphern}

Als Beispiel werden an dieser Stelle produktive Metaphern der Technik angeführt:

- Als Modell dient der menschliche oder tierische Körper.

- Als Modell dient die menschliche Psyche und Intelligenz.

- Als Modell dient die Alltagsmechanik (vgl. ROELCKE: 1999).

\subsection{Fachwortschatz}

Ein Fachwortschatz ist eine Menge kleiner bedeutungstragender und zugleich frei verwendbarer sprachlicher Einheiten (Fachwörter), die innerhalb der Kommunikation in einem bestimmten menschlichen Tätigkeitsbereich gebraucht werden. Daraus ergibt sich, dass es genaugenommen keine Fachsprache gibt, sondern so viele verschiedene Fachsprachen wie Fachrichtungen. Diese Fachsprachen dienen der Fachkommunikation über Fachinhalte - Gegenstände, Operationen, Prozesse, Verfahren, Theorien etc. - und benutzen dazu die sprachlich kürzeste und präziseste Form, nämlich die Fachtermini.

Die Fachsprache ist gebunden an:

- die Denkelemente des Faches, die aus den Fachtermini bestehen,

- die Denkstrukturen des Faches,

- die Mitteilungsstrukturen, die im Fach üblich sind (vgl. ROELCKE: 1999).

Eine Gliederung des Fachwortschatzes ist nach mehreren Kriterien möglich. ROELCKE (1999) berücksichtigt in seiner Kategorisierung die oft zu beobachtende 
enge Verflechtung des allgemeinsprachlichen und fachsprachlichen Wortschatzes und gliedert den Fachwortschatz in vier Gruppen:

- Die erste Gruppe bildet der intrafachliche Fachsprachwortschatz, der aus denjenigen Fachsprachwörtern besteht, die ausschließlich der betreffenden Fachsprache angehören.

- Die zweite Gruppe bildet der interfachliche Fachsprachwortschatz: Es handelt sich dabei um solche Fachwörter, die sowohl in dem betreffenden als auch in anderen fachsprachlichen Systemen erscheinen.

- Die dritte Gruppe besteht aus dem extrafachlichen Fachsprachwortschatz, also aus denjenigen Fachwörtern, die anderen fachsprachlichen Systemen zugehören, aber dennoch in Fachtexten des betreffenden Faches geäußert werden.

- Die vierte Gruppe umfasst den nichtfachlichen Fachsprachwortschatz dieser Texte, mit anderen Worten: die Menge der allgemeinen und fachlich nicht weiter geprägten Wörter, die trotzdem auch in Fachtexten verwendet werden.

Die oben angeführten Besonderheiten gelten im Allgemeinen für alle Fachsprachen. Im Folgenden soll den spezifischen Merkmalen der medizinischen Fachsprache nachgegangen werden.

\section{Besonderheiten der medizinischen Fachsprache}

Die medizinische Fachsprache kann man als eine berufsspezifische Sprache verstehen, die der Verständigung unter Fachkollegen, der Kommunikation bei der Behandlung menschlicher Krankheiten dient. Um die medizinischen Sachverhalte mitteilen zu können, muss der Lernende mit den sprachlichen Spezifika dieser Fachsprache bekannt gemacht werden. An dieser Stelle werden einige morphologisch-syntaktische Besonderheiten angeführt (siehe auch SCHRÖDER: 1988; BUHLMANN/ FEARNS: 2000). Hinsichtlich der morphologisch-syntaktischen Eigenschaften muss man sagen, dass es auch in der medizinischen Fachsprache keine eigene Grammatik gibt. Die linguistische Analyse zeigt trotzdem einige belegbare Besonderheiten, die man in Kürze folgendermaßen beschreiben kann:

In medizinischen Fachtexten findet man eine hohe Zahl an Substantiven. Sie sind dominierend und kommen am häufigsten im Singular mit dem bestimmten Artikel vor. Dativ und Akkusativ werden im Vergleich zur Allgemeinsprache seltener verwendet. In der medizinischen Fachsprache findet man spezifische Pluralkennzeichnungen, die in der Allgemeinsprache nicht gebräuchlich sind (z. B. Blut-Blute, Salz-Salze).

Auch Adjektive spielen in medizinischen Fachtexten eine wichtige Rolle. Sie dienen zur Präzisierung und Differenzierung der Benennungen. 
Im Bereich der Wortbildung sind die typischsten Wortbildungsarten der medizinischen Fachsprache Komposition und Derivation. Wortzusammensetzungen werden hauptsächlich von Substantiven gebildet (Pulsbeschleunigung, Kariesanfälligkeit), man findet dabei auch eine hohe Anzahl an Gliedern (Nasennebenhöhlenentzündung, Bandscheibenschäden, Krebsvorsorgeuntersuchung). Die Derivation dient oft zur Benennung von Geräten und Personen, am häufigsten kommt das Suffix -er vor (Kugelstopfer, Amalgamträger, Bohrer). Vertreten sind aber auch weitere Suffixe, wie z. B. -heit (Krankheit, Verwirrtheit), -keit (Flüssigkeit), -ung (Störung, Ablagerung), -bar (heilbar, vererbbar), -frei (zuckerfrei, kariesfrei), -los (schmerzlos), -ie (für Teilbereiche der Medizin: Psychiatrie, Immunologie, Chirurgie, Pädiatrie), -arm (fettarm, mineralienarm) oder -reich (vitaminreich, kalorienreich).

Einige Besonderheiten kann man auch im Bereich der Konversion finden, wo an erster Stelle die häufige Substantivierung von Infinitiven zu erwähnen ist (Löten, Bohren, Schmelzen). Charakteristisch sind auch konvertierte Namen, die aufgrund wissenschaftlicher und technischer Entdeckungen entstanden sind (Röntgen, Alzheimer, Parkinson). Von diesen Substantiven werden weiter Verben (röntgen) oder Adjektive (Parkinsonsche Krankheit) gebildet.

Außerdem muss noch betont werden, dass man in der medizinischen Fachsprache eine hohe Anzahl an Internationalismen lateinischen und griechischen Ursprungs findet (Gingivitis, Pulpa).

Eine interessante vergleichende Analyse der prozentualen Vertretung der einzelnen grammatischen Kategorien in Texten der Allgemeinsprache, Belletristik, Philosophie und Medizin bietet folgende Tabelle.

\begin{tabular}{|l|c|c|c|c|}
\hline & Gemeinsprache & Belletristik & Philosophie & Medizin \\
\hline Substantiv & $\mathbf{2 6 , 4}$ & $\mathbf{2 8 , 0}$ & 40,1 & $\mathbf{4 4 , 0}$ \\
\hline Adjektiv & $\mathbf{8 , 3}$ & 9,6 & 16,2 & $\mathbf{1 6 , 0}$ \\
\hline Adverb & 7,8 & 7,6 & 3,2 & 3,6 \\
\hline Verb & $\mathbf{1 9 , 5}$ & 19,5 & 11,4 & $\mathbf{1 0 , 5}$ \\
\hline Pronomen & 12,1 & 10,6 & 8,3 & 4,0 \\
\hline Konjunktion & 7,2 & 7,8 & 7,3 & 6,0 \\
\hline Präposition & 11,1 & 12,4 & 11,2 & 14,0 \\
\hline
\end{tabular}

(nach SCHRÖDER/SAARBECK: 2008)

Was die syntaktischen Besonderheiten der medizinischen Fachtexte angeht, sind hier auf Grund von Eindeutigkeit und Übersichtlichkeit größtenteils längere Sätze zu finden. Zu den meist vertretenen Nebensätzen gehören Relativsätze, Konditionalsätze (sehr oft ohne Einleitungswort wenn) und Nebensätze mit dass. 


\section{Kommunikativer und handlungsorientierter Fachsprachenunterricht}

Der Fachsprachenunterricht bildet gewissermaßen eine Brücke zwischen allgemeinsprachlichem Unterricht und Fachunterricht. Die Lernenden sollten auf der rezeptiven Ebene Fachtexte lesend verstehen, auf der produktiven Ebene sollten sie in der Lage sein, unterschiedliche Quellen für die Gestaltung eines Textes heranzuziehen, im Unterricht und in Übungssituationen Fragen zu stellen und komplexe Äußerungen zu produzieren. Um dies zu erreichen, sollten im Unterricht vor allem kompensatorische Strategien bei der Informationsentnahme und Textproduktion aufgebaut werden, die bestimmten stilistischen Eigentümlichkeiten der Kommunikation im Fach gerecht werden (Präzision, Differenziertheit, Hierarchisierung, Ökonomie etc.), Lesestrategien an den fachsprachlichen Texten entwickelt werden sowie morphologisch-syntaktische Besonderheiten der jeweiligen Fachsprache/Berufssprache thematisiert werden. Die Unterrichtsinhalte müssen der Forderung nach Authentizität und Relevanz für die (spätere) Berufspraxis der Lernenden entsprechen, der Ablauf des Unterrichts sollte gemäß moderner Unterrichtsprinzipien ${ }^{1}$ gestaltet sein. Von den Prinzipien, die für den modernen, lernerorientierten fachsprachlichen und berufsorientierten Fremdsprachenunterricht von entscheidender Bedeutung sind, möchten wir an dieser Stelle zwei hervorheben: das Prinzip der Kommunikation und das Prinzip der Handlungsorientierung.

\section{Das Prinzip der Kommunikation}

Auch eine fach- und berufsorientierte Fremdsprache lernt man als Kommunikationsmedium nur dann, wenn sie ausdrücklich und genügend oft in dieser Funktion verwendet wird. Es ist daher nötig, im Fremdsprachenunterricht die Mittel und Wege zu thematisieren, die den Lernenden ermöglichen, in der Sprache handeln zu können (vgl. TIMM: 2005, 45).

\section{Das Prinzip der Handlungsorientierung}

Handlungsorientierung gehört zu den aktuellsten Unterrichtsprinzipien des Sprachenlernens und -lehrens. Es bezieht sich auf die didaktischen und geistigen Tätigkeiten der Lernenden im Unterricht. Handeln heißt dabei beobachten, vergleichen, bedenken, aber auch verändern und herstellen. JANK/MEYER (1991) bezeichnen die Handlungsorientierung als ein Unterrichtskonzept, das den Schülern einen handelnden Umgang mit den Lerngegenständen und -inhalten

1 TIMM (2005, 45ff.) spricht von zehn Prinzipien des modernen, zukunftsweisenden Fremdsprachenlernens und -lehrens, zu denen er zählt: das Prinzip der Mündlichkeit, das Prinzip der Kommunikation, das Prinzip der funktionalen Fremdsprachigkeit, das Prinzip des Übens, das generative Prinzip, das Prinzip der muttersprachlichen Vorleistung, das Prinzip der Individualisierung oder Lernerorientierung, das Prinzip der Selbsttätigkeit, das Prinzip der Relevanz und das Prinzip der emotionalen Sicherheit. 
des Unterrichts ermöglicht. Für den fachsprachlichen und berufsorientierten Fremdsprachenunterricht lässt sich das Prinzip der Handlungsorientierung wie folgt näher bestimmen:

- Handlungsorientierter Unterricht ist u. a. durch Ganzheitlichkeit charakterisiert. Lernen erfolgt nämlich ganzheitlich, d. h. nicht nur einseitig kognitiv, sondern mit „Kopf, Hand und Herz“ (Pestalozzi). Tun und Denken werden miteinander verknüpft. Gerade bei berufsbezogenem Unterricht findet man unter den Lernern öfter praktisch-anschauliche Lerntypen, die eher auf das Tun gerichtet sind. Die Unterrichtsinhalte sollten nicht ausschließlich durch eine eindimensionale Fachsystematik bestimmt werden. Im Fremdsprachenunterricht geht es dann z. B. nicht allein um die grammatische Progression, sondern um die Bearbeitung des Themas aus der komplexen (Berufs-)Wirklichkeit.

- Handlungsorientierter Unterricht ist schüleraktiv und schülerorientiert. Der Lehrende versucht zu bewerkstelligen, dass die Lernenden möglichst viel erkunden, erproben, entdecken, erörtern, planen und verwerfen. Je mehr Handlungskompetenz der Lehrer vermittelt, desto selbstständiger können die Schüler weiterarbeiten.

- Ein weiteres Merkmal des handlungsorientierten Fremdsprachenunterrichts ist die Produktorientierung. Im Mittelpunkt des Unterrichts steht ein verwertbares Ergebnis, ein Handlungsprodukt. Diese Produkte können inszeniert (Rollenspiel, Planspiel usw.) oder hergestellt werden (Plakat, Wandzeitung, Collage, Flugschrift usw.). Das Produkt hat einen Gebrauchswert, es wird anderen vorgestellt, von ihnen auch ausgewertet und reflektiert.

- Der handlungsorientierte Unterricht legt großen Wert auf die Lern prozesse. Die Lernenden müssen im Team planen, erörtern, Entscheidungen treffen und sie auch erproben (learning by doing). So bauen sie fachliche, methodische und soziale Kompetenzen auf, die später bei der Berufsausübung benötigt werden können.

\subsection{Handlungsorientierte Methoden im berufsbezogenen und fachsprachlichen Deutschunterricht}

Sowohl in der Fachliteratur als auch in der Unterrichtspraxis findet man zahlreiche Methoden, die den Prinzipien der Handlungsorientierung entsprechen. Die Vorteile von solchen Methoden bestehen grundlegend darin, dass sie die Lernenden aktivieren und motivieren, ganzheitliches Lernen sowie selbstständiges, eigenverantwortliches Arbeiten und problemlösendes Verhalten ermöglichen, zu Kommunikation und Kooperation anregen und das Erlernen von Lern- und Arbeitstechniken und den Erwerb von Qualifikationen stimulieren. Einen sehr gut strukturierten Abriss für handlungsorientierte Methoden bieten HOFFMANN/LANGENFELD (2001), die diese Methoden in vier Gruppen einteilen: 
a) Methoden zur Schaffung von persönlicher und sachlicher Orientierung (z. B. Kennenlernspiele/Warming-up-Methoden, Selbstvorstellung, Partnervorstellung, Selbstporträt, Steckbrief).

b) Methoden zur Problemfindung (z. B. ABC-Liste, Aquarium, Brainstorming, Brainwriting, Kopfstand, Mind Map, Rollenspiel, Pro und Contra, Vortrag/ Referat).

c) Methoden zur Problemlösung (z. B. Betriebsbesichtigung, Betriebserkundung, Expertenbefragung,Einzelarbeit/Partnerarbeit/Gruppenarbeit,(Gruppen-)Puzzle, Fallbeispiel, Lernstationen/Stationenlernen, Leittext, Metaplan, Projekt).

d) Methoden zur Wissenssicherung, Reflexion, zum Transfer (z. B. ABC-Liste, Mind Map, Rollenspiel, Schülervortrag, Blitzlicht).

\subsection{Ein Unterrichtsentwurf für den medizinischen Fachsprachenunterricht}

Im Folgenden wird ein Unterrichtsverfahren aus SOJÁK (2004) vorgestellt, das sich als Hauptziel gesteckt hat, die Fachkommunikation zu entwickeln sowie die grammatischen Regularitäten zu reflektieren.

\section{Fall Hans}

Ein 14-jähriger Junge wurde wegen "Schwierigkeiten in der Schule und zu Hause" im Krankenhaus aufgenommen. Der Patient war zweites Kind der Familie. Die Mutter war bei seiner Geburt 29 Jahre alt gewesen. Der Schwangerschaftsverlauf war bis auf wiederholte Übelkeit und Erbrechen im 2. Schwangerschaftsmonat und einen Harnwegsinfekt im 6. SSM unauffällig gewesen. Wehen, Geburt und Körpergröße des Neugeborenen waren normal gewesen. Es lagen keine körperlichen Missbildungen des Neugeborenen vor. Die frühe motorische, psychische und geistige Entwicklung war regelrecht (Hochheben des Kopfes, allein sitzen, Worte sprechen, allein laufen). Das Kind war umgänglich und kam gut mit anderen zurecht. Nach der Geburt hatte die Mutter ihre Tätigkeit als Sekretärin aufgegeben, um sich seiner Erziehung zu widmen. Er trank gut (Muttermilch) und war später ein guter Esser. Er hatte keine zusätzlichen Vitamingaben erhalten. Er wurde regelmäßig geimpft und untersucht, ohne auffällige Befunde. Spezielle ansteckende Krankheiten lagen nicht vor. Es bestanden keine Schlafstörungen oder ungewähnliche Angewohnheiten während der Kleinkinderzeit oder der Kindheit. Der Junge entwickelte sich gut in der Schule und erreichte sehr gute und gute Noten bis zum Alter von 10 Jahren. Danach begann seine Leistung abzunehmen. Er fühlte sich nicht fit, um an sportlicher Aktivität teilzunehmen. Gelegentlich schienen sich seine Arme und Beine zu versteifen. Er klagte über Kopfschmerzen und Konzentrationsmangel während der Schulzeit. Er musste das 8. Schuljahr wiederholen, obwohl er zu diesem Zeitpunkt schon Nachhilfeunterricht erhielt. Er schien oft kein Interesse oder Motivation für irgendetwas zu haben. Er wurde immer geistesabwesender. Er brach alle Freundschaften ab. Er verbrachte die meiste Zeit zu Hause vor dem Fernseher. Es schien ihm egal zu 
sein, was er sah. Gelegentlich war er auch stublinkontinent. Manchmal brach er in plötzliches unbegründetes Brüllen aus, oder er drehte plötzlich seinen Kopf auf die Seite und streckte das Kinn vor. Verfolgungswahn oder suizidale Absichten traten nicht auf. Sein Appetit nahm zu und er wurde übergewichtig. Manchmal schob er Nahrung in seine Backen und schluckte sie dann einige Stunde lang nicht herunter. Dann wieder hatte er Schluckschwierigkeiten.

\section{Diagnose:}

Wilson-Erkrankung mit der Hauptmanifestation einer Frontalhirndemenz.

\section{Unterrichtsverfahren}

\section{a) Kommunikative Übungen:}

Für kommunikative Übungen wurden zwei Methoden kombiniert: der modifizierte Metaplan ${ }^{1}$ und das Rollenspiel. Das Unterrichtsgeschehen verläuft in folgenden Phasen:

- Die Schüler arbeiten in Paaren; sie lesen den Text, wobei sie auf die vom Lehrer vorbereiteten Karten stichwortartig die Diagnose von Hans im Einzelnen aufschreiben:

\begin{tabular}{|c|c|c|}
\hline $\begin{array}{l}\text { Schwangerschaftsverlauf } \\
\text { der Mutter } \\
\ldots \ldots \ldots \ldots \ldots \\
\ldots \ldots \ldots \ldots \ldots\end{array}$ & $\begin{array}{l}\text { Die frühe Entwicklung } \\
\text { des Kindes } \\
\\
\ldots \ldots \ldots \ldots \ldots \\
\ldots \ldots \ldots \ldots \ldots\end{array}$ & $\begin{array}{l}\text { Ergebnisse der ärztlichen } \\
\text { Untersuchungen in der } \\
\text { Kleinkinderzeit und der Kindheit } \\
\ldots \ldots \ldots \ldots \ldots \\
\ldots \ldots \ldots \ldots \ldots\end{array}$ \\
\hline $\begin{array}{l}\text { Leistungen in der Schule } \\
\text { vor der Erkrankung }\end{array}$ & Mutterpflege & $\begin{array}{l}\text { Leistungen in der Schule nach der } \\
\text { Erkrankung }\end{array}$ \\
\hline $\begin{array}{l}\ldots \ldots \ldots \ldots \ldots \ldots \\
\ldots \ldots \ldots \ldots \ldots \ldots\end{array}$ & $\begin{array}{l}\ldots \ldots \ldots \ldots \ldots \ldots \\
\ldots \ldots \ldots \ldots \ldots \ldots\end{array}$ & $\ldots+\ldots+\ldots, \ldots$ \\
\hline
\end{tabular}

1 Nach HOFFMANN/LANGENFELD (2001) besteht die didaktische Rolle des Metaplans/ der Moderationsmethode darin, die aktive Beteiligung aller Lernenden am Arbeitsprozess zu fördern, die Kommunikation, Kooperation, Kreativität und Interaktion zu entwickeln und die Motivation sowie die Merkfähigkeit durch Tun und Visualisierung zu erhöhen.

Es handelt sich um ein themenübergreifendes Verfahren:

- Einstieg: Die Ziele werden formuliert und die Methodik geklärt.

- Themenorientierung: Das Thema/Problem wird in Form einer präzisen Frage festgelegt. Die Karten werden verteilt. Die Ideen werden gesammelt und auf Kärtchen geschrieben (1 Karte - 1 Idee). Die Karten werden für alle sichtbar an die Pinnwand geheftet und die Ideen ,geclustert' (nach inhaltlichen Schwerpunkten geordnet).

- Themenordnung: Ein Themenspeicher wird angelegt. Die Themen werden ,gewichtet', d. h. die Schüler geben mit Hilfe von Klebepunkten an, in welcher Reihenfolge die Themen bearbeitet werden sollen.

- Themenbearbeitung: Das Thema wird in Kleingruppen diskutiert, bearbeitet und für die Präsentation vorbereitet.

- Ergebnissicherung: Die Ergebnisse werden im Plenum vorgestellt und zusammengefasst.

- Abschluss: Der Gruppenprozess wird reflektiert. 


\begin{tabular}{|l|l|l|}
\hline $\begin{array}{l}\text { Psychische Symptome der } \\
\text { Erkrankung }\end{array}$ & $\begin{array}{l}\text { Physische Symptome der } \\
\text { Erkrankung }\end{array}$ & \\
$\ldots \ldots \ldots \ldots \ldots$ & $\ldots \ldots \ldots \ldots \ldots$ & \\
$\ldots \ldots \ldots \ldots \ldots$ & $\ldots \ldots \ldots \ldots \ldots$ & \\
\hline
\end{tabular}

- Die Karten mit Informationen werden an die Tafel (Pinnwand) geheftet und strukturiert (z. B. 1. Mutter und Schwangerschaft, 2. Probleme in der Schule 3. psychische Schwierigkeiten und physische Schwierigkeiten).

- Es werden vier Expertenteams gebildet (Schulpädagogen, Frauenärzte, Psychologen und Psychiater) und ein Primarius gewählt, der die Fachdiskussion leitet und moderiert.

- Die Vertreter der einzelnen Expertenteams berichten mündlich über die einzelnen Aspekte der Diagnose von Hans, entwerfen eine mögliche Therapie aus der Sicht der jeweiligen Expertengruppe und stellen sie im Plenum vor.

- Der Moderator (Primarius) sorgt dafür, dass alle Vorschläge diskutiert bzw. kommentiert werden.

\section{b) Grammatische Übungen: ${ }^{1}$}

1. Unterstreichen Sie alle Verbformen, die im Text im Präteritum vorkommen und finden Sie die richtigen Infinitivformen.

2. Finden Sie im Text die Infinitivkonstruktionen mit $u m \ldots z u$ und bilden Sie Nebensätze mit der entsprechenden Konjunktion.

\section{Zusammenfassung:}

Sprachkenntnisse weisen heutzutage einen hohen Stellenwert auf, wobei auch das Lehren und Lernen von Fremdsprachen ein anderes Bild als früher bietet. Die neuen Herausforderungen für den Fremdsprachenunterricht rühren aus den politischen und gesellschaftlichen Veränderungen in Europa sowie der weiter zunehmenden Verflechtung von Wirtschafts-, Kultur- und Lebensräumen her. $\mathrm{Zu}$ den bedeutendsten Merkmalen des Fremdsprachenunterrichts gehört ein wesentliches Anwachsen des fachsprachlichen und berufsorientierten Sprachunterrichts, der u. a. für die Mobilität auf dem internationalen Arbeitsmarkt von herausragender Bedeutung ist. Um diesen Unterricht effizient gestalten zu können, braucht man nicht nur geeignete Unterrichtsmaterialien, sondern auch qualifizierte Lehrkräfte, die sich dessen bewusst sind, dass eine Fach-/Berufssprache als Kommunikationsmittel im Zuge der der Sozialisation innerhalb einer bestimmten Disziplin, eines bestimmten Faches oder Berufs zu lernen ist.

Schlüsselwörter:

Fremdsprachenunterricht - fachsprachlicher Sprachenunterricht - berufsorientierter Sprachenunterricht - Mobilität auf dem Arbeitsmarkt - sprachdidaktisch

1 Siehe auch die an der Pädagogischen Fakultät der Masaryk-Universität erstellte Abschlussarbeit KOČOVÁ (2008). 


\section{Literatur:}

BUHLMANN/FEARNS (2000): Buhlmann, Rosemarie/Fearns, Anneliese. Handbuch des Fachsprachenunterrichts unter besonderer Berücksichtigung naturwissenschaftlich-technischer Fachsprachen. (Neubearbeitung, ältere Auflagen bei Langenscheidt). Tübingen: Narr Verlag, 2000.

BUTZKAMM (2005): Butzkamm, Wolfgang. Zehn Prinzipien des Fremdsprachenlernens und -lehrens, in: Timm, Johannes-Peter (Hg.): Englisch lernen und lehren. Didaktik des Englischunterrichts. Berlin: Cornelsen, 2005, 45-52.

CHRIST(2002): Christ, Herbert. Fremdsprachenunterricht in neuen Dimensionen im 21. Jahrhundert, in: Neveling, Christiane (Hg.): Perspektiven für die zukünftige Fremdsprachendidaktik. Tübingen: Gunter Narr Verlag, 2002, 25-44.

HOFFMANN/LANGENFELD (2001): Hoffmann, Bärbel/Langenfeld, Ulrich. Methodenmix. Unterrichtliche Methoden zur Vermittlung beruflicher Handlungskompetenz in kaufmännischen Fächern. Darmstadt: Winklers Verlag, 2001.

JANÍKOVÁ (2005): Janíková, Vèra. Didaktik des Deutschen als Fremdsprache. Anmerkungen zu aktuellen Themen mit Aufgaben zum Selbststudium. Brno: Masarykova univerzita, 2005.

JANK/MEYER (1994): Jank, Werner/Meyer, Hilbert. Didaktische Modelle. Berlin: Cornelsen, 1994.

KOČOVÁ (2008): Kočová, Kateřina. Aneignung der Grammatik im Deutschunterricht an der medizinischen Fachoberschule. Abschlussarbeit. Pädagogische Fakultät der MasarykUniversität, Ms., 2008.

ROELCKE (1999): Roelcke, Thorsten. Fachsprachen. Berlin: Erich Schmidt Verlag, 1999.

SCHRÖDER (1988): Schröder, Hartmut. Aspekte einer Didaktik/Methodik des fachbezogenen Fremdsprachenunterrichts (Deutsch als Fremdsprache). Unter besonderer Berücksichtigung sozialwissenschaftlicher Fachtexte. (Werkstattsreihe DaF 20). Frankfurt a. M. etc.: Peter Lang, 1988.

SCHRÖDER/SAARBECK (2008): Schröder, Hartmut/Saarbeck, Ursula. Didaktik des fachbezogenen Fremdsprachenunterrichts. URL: http://www.sw2.euv-frankfurt-o.de/ ViruLearn/dff/21_april_c.html [abgerufen am 5.5.2008].

SOJÁK (2004): Soják, Karel. Nèmčina pro vyšš́ zdravotni školy a bakalárské studium. Praha: Eurolex Bohemia, 2004. 